\section{Kolloidosmotischer Druck}

O. Müller-Plathe

Hamburg, Deutschland

Synonym(e) Onkotischer Druck; KOD

Englischer Begriff colloid osmotic pressure

Definition Der kolloidosmotische Druck (KOD) ist der Anteil des osmotischen Drucks, der von den kolloidal gelösten, nicht diffusiblen Makromolekülen des Plasmas, vorzugsweise $\triangleright$ Albumin, verursacht wird.

Beschreibung Die Proteine bewirken den kolloidosmotischen Druck von etwa $25 \mathrm{mmHg}(3,3 \mathrm{kPa})$ zum einen als Solute mit einer Konzentration von ca. $1 \mathrm{mmol} / \mathrm{L}$, zum anderen über eine Donnan-Verschiebung von Ionen ( $\triangleright$ Donnan-Gleichgewicht). Der KOD ist im Kapillarbereich als Gegenspieler des dort herrschenden hydrostatischen Drucks maßgeblich an der Regulierung des Wasseraustauschs mit dem Interstitium beteiligt. Ein zu geringer KOD begünstigt bei unverändertem oder erhöhtem hydrostatischen Druck den Wasseraustritt in den extravasalen Raum (Gewebsödeme, Lungenödem). Die Messung des KOD wird zur Kontrolle der postoperativen Flüssigkeitstherapie genutzt. Sie ist darin, weil sie neben den Proteinen auch die Wirkung großmolekularer Plasmaexpander für die Erhaltung des intravasalen Volumens erfasst, der Messung von Gesamtprotein oder Albumin überlegen.

Methode: Das Onkometer besteht aus zwei durch eine semipermeable Membran getrennte Kammern, deren eine die Plasmaprobe aufnimmt, während die andere als Referenzzelle physiologische Kochsalzlösung enthält und mit einem Drucksensor ausgestattet ist. Dieser erfasst die durch die Kolloide ausgelöste Flüssigkeitsverschiebung als Druckabfall in der Referenzzelle.

Referenzbereich - Erwachsene $20-35 \mathrm{mmHg} ; 2,74,7 \mathrm{kPa}$.

\section{Literatur}

Kaminski MV, Haase TJ (1992) Albumin and colloid osmotic pressure. Implications for fluid resuscitation. Crit Care Clin 8:311-321 